\title{
SOLUBLE BOLTZMANN EQUATIONS FOR INTERNAL STATE AND MAXWELL MODELS
}

\author{
E. FUTCHER and M.R. HOARE \\ Department of Physics, Bedford College, University of London, London, England \\ and \\ E.M. HENDRIKS and M.H. ERNST \\ Instituut voor Theoretische Fysica, Rijksuniversiteit Utrecht, Utrecht, The Netherlands
}

Received 13 November 1979

\begin{abstract}
We consider a class of scalar nonlinear Boltzmann equations describing the evolution of a microcanonical ensemble in which sub-systems exchange internal energy 'randomly' in binary interactions.

In the continuous variable version these models can equally be interpreted as Boltzmann equations for Maxwell type molecules in arbitrary dimensionality.

We construct general solutions in the form of a Fourier series; the expansion coefficients (Sonine or Meixner moments) satisfy the same recursive system of coupled equations as the ordinary moments, and can be solved sequentially.
\end{abstract}

\section{Introduction}

In recent years increasing attention has been turned to the search for exact solutions of nonlinear Boltzmann equations. Whilst the possibility of exact solutions for so-called 'Maxwell molecules' has been in the air for some time (see e.g. Truesdell')), most stimulus has undoubtedly come from the work of Bobylev $^{2,3}$ ), Krook and $\mathrm{Wu}^{4}$ ) who obtained an exact (similarity) solution (the BKW solution) to the Boltzmann equation for Maxwell molecules in a closed, spatially-homogeneous system. Various applications of similarity solutions and extensions to related models have been considered in the literature ${ }^{5-9}$ ).

Our primary objective is to construct general solutions of nonlinear kinetic equations for our models by Fourier expansion. The method has been introduced by $\mathrm{Kac}^{10}$ ) for a special model Boltzmann equation, and extended by Ernst ${ }^{8}$ ) to a large class of Maxwell-type models. The previous results for a few special models, all contained in ref. 8 , were rediscovered recently by Barnsley, Turchetti and Cornille ${ }^{11-13}$ ), and extended to nonisotropic velocity distributions by Weinert et al. ${ }^{14}$ ). Here we make clear the relevance of these results to the relaxation of internal (e.g. vibrational) states and expose their 
analogues in a discrete state-variable. This program is thus to an extent a synthesis of the recent work of Cooper, Hoare and Rahman ${ }^{15}$ ) on distributive energy transfer between internally-structured molecules with the more traditional kinetic-theory approach. We conclude with a brief investigation of the linear approximations to our models which are shown to lead to exact solutions equivalent in effect to the Cooper-Hoare-Rahman solutions for linear distributive processes.

We are interested here in a general type of model Boltzmann equations for the evolution of a distribution function, $P(x, t)$, in a scalar quantity $x \in(0, \infty)$, globally conserved in a closed, microcanonical system. The most general equation we need to consider can be written in the form

$$
\begin{aligned}
\partial_{t} P(x, t)= & \iint \mathrm{d} u \mathrm{~d} y\{P(y, t) P(u-y, t) K(x, y ; u) \\
& -P(x, t) P(u-x, t) K(y, x ; u)\}
\end{aligned}
$$

whereby, as evident from the structure of the collision integral on the right, relaxation proceeds via binary interactions with molecular chaos, and is governed by a scattering kernel $K(x, y ; u)$ in which the total state-quantity $u$ available in a collision enters explicitly. In practice, however two types of simplification are usually made. Firstly, it is assumed that the scattering kernel may be replaced by one of the form $K(x ; u)$ whose dependence involves only the final state quantity $x$, and the total state quantity $u$; not the initial state $y$. In this way the equation reduces to

$$
\begin{aligned}
\partial_{t} P(x, t)= & \int_{x}^{\infty} \mathrm{d} u \int_{0}^{u} \mathrm{~d} y[P(y, t) P(u-y, t) K(x ; u) \\
& -P(x, t) P(u-x, t) K(y ; u)] .
\end{aligned}
$$

Secondly, the total rate of outscattered pairs $\int_{0}^{\infty} d y K(y ; u)$ is taken to be a constant $\omega$, independent of the initial state. The second term in (1.2) above then reduces to $-P(x, t)$ in the scaled time. Known solutions to equations of this form are virtually restricted to the Tjon-Wu model, for which $K(x ; u)$ is in effect the uniform distribution $\left.K(x ; u)=u^{-1}, 0 \leqslant x \leqslant u^{6,7}\right)$ and the ErnstHendriks model in which $\left.K(x ; u)=1^{16}\right)$. Other authors such as Rouse and Simons ${ }^{17}$ ), and Ray $^{18}$ ) have considered aspects of moment evolution and entropy production in the BKW and related models.

\section{Internal state models and Maxwell scattering}

Although in previous literature emphasis has invariably been on Maxwell molecules in translational motion, it is important to stress that eq. (1.1) and 
(1.2), particularly in their discrete variable formulations, can be applied equally well to the relaxation of internal state-variables such as vibrational energy. There is, in fact, a small literature on the relaxation of closed oscillator systems ${ }^{12-21}$ ) and the unification of this aspect of Boltzmann equation theory with the more traditional kinetic-theory approach and the treatment of other types of scalar transport equation (see e.g. the work of Melzak $^{22}$ ) could be said to be long overdue.

In this general context we shall consider what appears to be the first example of an exact solution to a nonlinear oscillator relaxation problem in which multiple internal degrees of freedom are allowed for and where a discrete or continuous state-space may be assumed with equal facility. We shall first consider the nature of the scattering kernels which arise from the hypothesis of random sharing of internal energy between degrees of freedom stressing the close parallel between the cases where the degrees of freedom involved are actually internal and where they are in effect the translational components for Maxwell molecules. We shall then go on to obtain solutions equally valid for the two cases, emphasizing to some extent the discrete formulation because this is less familiar and can always be used to yield the continuous results by a limiting process.

\section{Random scattering kernels}

The idea of random scattering between systems of multiple degrees of freedom has been developed by Cooper, Hoare and $\operatorname{Rahman}^{15}$ ) as a tool for modeling energy-transfer between complex systems interacting linearly with a heat bath and lends itself in slightly modified form to the treatment of binary scattering in a closed system. The essential idea is that the total energy available to a binary collision complex is redistributed with equal a priori probability over all energy microstates of the two partners. The same idea can also be applied to systems of structureless particles with $d$ translational degrees of freedom, in which binary collisions cause an initial pair state, specified by $\left(v, v_{1}\right)$ to scatter uniformly over the $2 d$-dimensional energy surface. With the additional assumption of a mean collision number independent of the energy it leads to a Boltzmann equation for Maxwell type molecules.

\subsection{Diffuse scattering between d-dimensional molecules}

We start with the more familiar case of the Boltzmann equation for a system of structureless particles with a spatially uniform velocity distribution, 
and we allow for $d$ translational degrees of freedom per particle. The Boltzmann equation can then be written as ${ }^{23}$ )

$$
\begin{gathered}
\partial_{f} f(v, t)=\iiint_{\left.-W\left(v^{\prime} v_{1}^{\prime} \mid v v_{1}\right) f(v, t) f\left(v_{1}, t\right)\right\},} \mathrm{d} v^{\prime} \mathrm{d} v_{1}^{\prime}\left\{W\left(v v_{1} \mid v^{\prime} v_{1}^{\prime}\right) f\left(v^{\prime}, t\right) f\left(v_{1}^{\prime}, t\right)\right. \\
\text {. }
\end{gathered}
$$

where $W\left(v^{\prime} v_{1}^{\prime} \mid v v_{1}\right)$ is the probability per unit time for a pair of particles with initial velocities $\left(v, v_{1}\right)$ to scatter into final velocities $\left(v^{\prime}, v_{1}^{\prime}\right)$. In general, $W$ will be proportional to the differential scattering cross section, and will contain $\delta$-functions restricting the final states to those conserving both energy as well as momentum. As a simplification to the general form (3.1) Tjon and $\mathrm{Wu}^{6}$ ) have introduced a class of models, where $W$ has the special form

$$
W\left(v^{\prime} v_{1}^{\prime} \mid v v_{1}\right)=a(u) \delta\left(u-u^{\prime}\right)
$$

with $a(u)$ an (as yet) arbitrary function of the total energy $u=\frac{1}{2} v^{2}+\frac{1}{2} v_{1}^{2}$. Here the scattered states $\left(v^{\prime}, v^{\prime}\right)$ are uniformly distributed over a $2 d$-dimensional sphere with $\left(v^{\prime}\right)^{2}+\left(v_{1}^{\prime}\right)^{2}=v^{2}+v_{1}^{2}$, i.e. in the microscopic scattering laws energy is conserved, but momentum is uninterpreted. Model Boltzmann equations violating momentum conservation have also been considered by e.g. $\mathrm{McKean}^{24}$ ) and $\left.\mathrm{Kac}^{10}\right)$. The function $a(u)$ is determined by the further simplifying assumption that for any state $\left(v, v_{1}\right)$ the total rate of scattered pairs is independent of the total energy. Thus we have:

$$
\int \mathrm{d} v^{\prime} \mathrm{d} v_{1}^{\prime} W\left(v^{\prime} v_{1}^{\prime} \mid v v_{1}\right)=\Omega_{2 d}(2 u)^{d-1} a(u)=\text { constant }=1,
$$

where $\Omega_{d}=2 \pi^{d / 2} / \Gamma(d / 2)$ is the surface of a $d$-dimensional sphere. With this choice of $a(u)$ the Boltzmann equation takes the standard form

$$
\partial_{f} f(v, t)=\int \mathrm{d} v_{1} \int_{0}^{\pi} \mathrm{d} \chi(\sin \chi)^{d-1} A\left[f\left(v^{\prime}, t\right) f\left(v_{1}^{\prime}, t\right)-f(v, t) f\left(v_{1}, t\right)\right]
$$

and the collision dynamics is

$$
\begin{aligned}
& v^{\prime}=\left\{v^{2}+v_{1}^{2}\right\}^{1 / 2} \cos \left(\frac{1}{2} \chi\right), \\
& v_{1}^{\prime}=\left\{v^{2}+v_{1}^{2}\right\}^{1 / 2} \sin \left(\frac{1}{2} \chi\right),
\end{aligned}
$$

where $\chi$ is the formal analogue of the scattering angle and $(\sin \chi)^{d-1} \mathrm{~d} \chi$ is proportional to the infinitesimal solid angle in $d$ dimensions. In the standard form of the Boltzmann equation appears the combination $g I(g, \chi)$, the product of the relative velocity $g=\left|v-v_{1}\right|$ and the differential scattering cross section $I(g, \chi)$. The analogue of this combination is the constant $A$, which makes this model a model for $d$-dimensional Maxwell molecules with diffuse scattering. 
It is only well defined for isotropic velocity distributions $f(v, t)=f(v, t)$. Hence it is convenient to introduce the energy distribution function $P(x, t)$ with $x=\frac{1}{2} v^{2}$, defined as:

$$
P(x, t) \mathrm{d} x=f(v, t) \mathrm{d} v=f(v, t) \Omega_{d}(2 x)^{d / 2-1} \mathrm{~d} x .
$$

The Boltzmann equation now takes the form

$$
\begin{aligned}
\partial_{t} P(x, t)= & \iiint \mathrm{d} x_{1} \mathrm{~d} x^{\prime} \mathrm{d} x_{\{}^{\prime}\left\{w\left(x x_{1} \mid x^{\prime} x_{1}^{\prime}\right) P\left(x^{\prime}, t\right) P\left(x_{1}^{\prime}, t\right)\right. \\
& \left.-w\left(x^{\prime} x_{1}^{\prime} \mid x x_{1}\right) P(x, t) P\left(x_{1}, t\right)\right\},
\end{aligned}
$$

where all $x$-integrals extend from 0 to $\infty$. The transition probability can be obtained from (3.2) and (3.3) as

$$
\begin{aligned}
w\left(x^{\prime} x_{1}^{\prime} \mid x x_{1}\right) & =\Omega_{d(2 x)^{2 / 2-1}\left(2 x_{1}\right)^{d / 2-1} W\left(v^{\prime} v_{1}^{\prime} \mid v v_{1}\right)} \\
& =W_{p, p}\left(x, x+x_{1}\right) \delta\left(x+x_{1}-x^{\prime}-x_{1}^{\prime}\right)
\end{aligned}
$$

with $p=\frac{1}{2} d$, and $W_{p, p}(x, u)$ is the symmetric Beta distribution

$$
W_{p, p}(x ; u)=\frac{x^{p-1}(u-x)^{p-1}}{B(p, p) u^{2 p-1}}
$$

where $B(p, q)=\Gamma(p) \Gamma(q) / \Gamma(p+q)$ is the Beta function. We see that (3.7) reduces to (1.2), $W_{p, p}\left(x, x+x_{1}\right)$ being the number of pair states in which one particle has an energy in $\mathrm{d} x$ about $x$, and the other in $\mathrm{d} x_{1}$ about $x_{1}$ to the number of pair states with energy in $\mathrm{d} u$ about $x+x_{1}=u$. The function (3.9) integrates to unity over the interval $x \in(0, u)$ by the Beta-function integral

$$
\int_{0}^{u} W_{p, p}(x ; u) \mathrm{d} x=1
$$

and has the necessary interactional symmetry $W(x ; u)=W(u-x ; u)$. For later reference we quote the moments of the symmetrical Beta distribution,

$$
m^{(n)}(u)=\int_{0}^{u} x^{n} W_{p, p}(x ; u) \mathrm{d} x=\frac{(p)_{n} u^{n}}{(2 p)_{n}},
$$

where $(p)_{n}$ is the Pochhammer (rising factorial) function: $(p)_{n}=$ $p(p+1)(p+2) \ldots(p+n-1)$. As obvious by symmetry: $m^{(1)}(u)=\frac{1}{2} u$. The second moment is $m^{(2)}(u)=\frac{1}{2} u^{2}(p+1) /(2 p+1)$.

\subsection{Continuous and discrete random scattering between p-molecules}

For identical sub-systems each of $p$ degrees of freedom the required scattering kernel $K(x ; u)$ is simply a symmetrical Beta distribution, as follows 
on performing the necessary volume integrals over the energy subspaces ${ }^{15 a}$ ). Thus specifying this as the probability density

$$
\begin{gathered}
W_{p, p}(x ; u) \mathrm{d} x=\operatorname{Pr}\{\text { One } p \text {-molecule in complex has energy in } \mathrm{d} x \text { about } \\
x \mid \text { two in complex have energy } u\} .
\end{gathered}
$$

This interpretation is clearly analogous to (3.9). The above analysis shows that models involving higher-dimensional Maxwell scattering can equally well be treated in terms of random scattering from internal states, provided that the latter is interpreted if necessary to allow the half-integer degrees of freedom $p=\frac{1}{2} d$.

The discrete analogue of (3.9) on the state-space of positive integers $i \in[0,1, \ldots k]$ is the symmetrical Negative Hypergeometric (NHG) distribution:

$$
\begin{aligned}
W_{p, p}(i ; k)= & \operatorname{Pr}\{\text { One } p \text {-molecule in complex has } i \text { quanta } \mid \text { two in complex } \\
& \text { have } k \text { quanta }\} \\
= & \frac{(i+1)_{p-1}(k-i+1)_{p-1}}{B(p, p)(k+1)_{2 p-1}}
\end{aligned}
$$

provided that the degrees of freedom involved are degenerate and the quanta have equal energy $\epsilon_{0}=h \nu$. The derivation ${ }^{15 c}$ ) now turns upon simple combinatorics of "balls" (quanta) in "boxes" (degrees of freedom or vibrational modes). We may note that the use of the Pochhammer notation in (3.13) brings out the clear analogy between the discrete and continuous distributions. The normalization

$$
\sum_{i=0}^{k} W_{p, p}(i ; k)=1
$$

and the factorial moments are

$$
\bar{m}^{(n)}(k)=\sum_{i=n}^{k} i^{(n)} W_{p, p}(i ; k)=\frac{(p)_{n} k^{(n)}}{(2 p)_{n}}
$$

in which $k^{(n)}$ is now the falling-factorial function: $k^{(n)}=$ $k(k-1)(k-2) \ldots(k-n+1)$. The first and second of these are $m^{(1)}(k)=\frac{1}{2} k$, $m^{(2)}(k)=\frac{1}{2} k(k-1)(p+1) /(2 p+1)$. In deriving (3.14) and (3.15) we used Vandermonde's theorem, the finite-difference extension of the binomial theorem. It can be written in many forms. We shall require all three of the following in this paper:

$$
\begin{aligned}
& \sum_{i=0}^{N}(i+1)_{\alpha}(N-i+1)_{\beta}=(N+1)_{\alpha+\beta+1} \frac{\Gamma(\alpha+\beta+1)}{\Gamma(\alpha+1) \Gamma(\beta+1)}, \\
& \sum_{i=0}^{N}\left(\begin{array}{c}
N \\
i
\end{array}\right) \alpha^{(i)} \beta^{(N-i)}=(\alpha+\beta)^{(N)},
\end{aligned}
$$




$$
\sum_{i=0}^{N}\left(\begin{array}{c}
N \\
i
\end{array}\right)(\alpha)_{i}(\beta)_{N-i}=(\alpha+\beta)_{N}
$$

The above are valid for non-integral $\alpha$ and $\beta$ provided the factorial functions can be replaced by finite values of the corresponding Gamma functions.

The limiting property which takes $W_{p, p}(i ; k)$ into the probability density $W_{p, p}(x, u)$ is of fundamental importance throughout this paper. It is defined as

$$
i \rightarrow \infty, \quad \epsilon_{0} \rightarrow 0 \quad \text { with } i \epsilon_{0}=x=\text { finite }
$$

with $\Delta i=\mathrm{d} x / \epsilon_{0}$. Hence, putting $k=u / \epsilon_{0}$ and using the relation $\lim \epsilon_{0}^{p}(i)_{p}=x^{p}$ yields the desired result

$$
\lim W_{p, p}(i ; k) \Delta i=\lim W_{p, p}\left(\frac{x}{\epsilon_{0}} ; \frac{u}{\epsilon_{0}}\right) \frac{\mathrm{d} x}{\epsilon_{0}}=W_{p, p}(x ; u) \mathrm{d} x
$$

\section{Kinetic equations}

With only a scalar state-variable involved, the relaxation of a closed set of sub-systems in binary interaction will, in the case of continuous systems at constant collision rate, be governed by an equation of type (1.2). This we may now write somewhat more explicitly as

$$
\left(\partial_{t}+1\right) P(x, t)=\int_{x}^{\infty} \mathrm{d} u \int_{0}^{u} \mathrm{~d} y P(y, t) P(u-y, t) W_{p, p}(x ; u)
$$

with $W_{p, p}(x, u)$ the Beta distribution of eq. (3.9), and time scaled to unit collision frequency.

The discrete analogue of the above is easily written

$$
\left(\partial_{t}+1\right) P(i, t)=\sum_{k=i}^{\infty} \sum_{j=0}^{k} P(j, t) P(k-j, t) W_{p, p}(i ; k)
$$

with $W_{p, p}(i, k)$ now the NHG distribution of eq. (3.13). We note that it is natural, and indeed essential to index from the state $i=0$ corresponding to the ground or 'empty' state of the particles. Several alternative forms of the above equations are possible on shifting the summation variables.

It is implicit in our whole program that the above equations are to be solved under the specification of a total energy, which we can express as a mean energy per degree of freedom $\epsilon$ in either case and a fundamental quantum unit $\epsilon_{0}$ in the discrete case.

While the main object of attention will usually be the distribution function, the moments of the same will also be shown to be of central importance in 
determining this. These we shall define as

$$
m_{n}(t)=\int_{0}^{\infty} x^{n} P(x, t) \mathrm{d} x
$$

and

$$
\bar{m}_{n}(t)=\sum_{i=n}^{\infty} i^{(n)} P(i, t)
$$

the latter being the factorial moment for the discrete distribution. The continuous limit (3.19) yields

$$
\lim \epsilon_{0}^{n} \bar{m}_{n}=m_{n}
$$

In some sections, however, it will prove an advantage to work with the modified forms: $\mu_{n}=m_{n} / n$ ! and $\bar{\mu}_{n}=\bar{m}_{n} / n$ ! Furthermore, the total number of particles and total energy are conserved, i.e.

$$
\begin{array}{lll}
m_{0}(t)=1 & m_{1}(t)=p \epsilon \\
& \text { or } & \\
\bar{m}_{0}(t)=1 & \bar{m}_{1}(t)=p \epsilon / \epsilon_{0},
\end{array}
$$

as follows from the symmetry $K(x, u)=K(u-x, u)$ without reference to a particular form of $K(x, u)$.

\section{Equilibrium}

On physical grounds we would expect the solutions of either (4.1) or (4.2) to tend to a unique stationary distribution $\boldsymbol{P}_{0}(x)$ or $\boldsymbol{P}_{0}(i)$ as $t \rightarrow \infty$. A satisfactory account of this process would nevertheless require that we first demonstrate the stationarity of $\boldsymbol{P}_{0}$ in either case, then provide an $\boldsymbol{H}$-theorem to guarantee evolution to this form.

Given particular kernels $W_{p, p}$ it requires only commonplace textbook arguments to supply the necessary stationary solutions. A brief summary seems justified here because of the less usual context and because the derivation is not particularly familiar in the case of the discrete variable. Taking the continuous variable case first, we note from eq. (1.2) that a sufficient condition for $P_{0}$ to be stationary is that it satisfies the detailed balance condition

$$
P_{0}(x) P_{0}(u-x) K(y ; u)=P_{0}(y) P_{0}(u-y) K(x ; u) .
$$


With $K(x ; u)$ given by the Beta distribution (3.9) this condition is clearly met by

$$
P_{0}(x)=a x^{p-1} c^{x}
$$

with constants $a$ and $c<1$ to be determined by the conservation of number and total energy respectively. With

$$
\int_{0}^{\infty} \mathrm{d} x P_{0}(x)=1 ; \quad \int_{0}^{\infty} \mathrm{d} x x P_{0}(x)=p \epsilon
$$

we find immediately that

$$
P_{0}(x)=\Gamma(p)^{-1} \epsilon^{-p} x^{p-1} \mathrm{e}^{-x / \epsilon} .
$$

This is a Gamma distribution with mean $\epsilon$. The higher moments, which we shall need in later sections, may be written in the form

$$
m_{n}(\infty)=\int_{0}^{\infty} x^{n} P_{0}(x) \mathrm{d} x=(p)_{n} \epsilon^{n}
$$

For the discrete variable the derivation is essentially parallel. We see that for stationarity

$$
\frac{P_{0}(j) P_{0}(k-j)}{P_{0}(i) P_{0}(k-i)}=\frac{(j+1)_{p-1}(k-j+1)_{p-1}}{(i+1)_{p-1}(k-i+1)_{p-1}}
$$

which is solved by $P_{0}(i)$ in the form

$$
P_{0}(i)=a(i+1)_{p-1} c^{i} .
$$

This time the constants $a$ and $c<1$ are determined by the binomial theorem:

$$
\sum_{i=0}^{\infty}(i+1)_{p-1} c^{i}=\Gamma(p)(1-c)^{-p} .
$$

Thus, by normalization $a=(1-c)^{p} / \Gamma(p)$. Applying now the energy condition it follows in similar fashion that

$$
p \epsilon=\epsilon_{0} \sum_{i=1}^{\infty} i P_{0}(i)=\epsilon_{0} p c /(1-c)
$$

and hence that $c=\epsilon /\left(\epsilon_{0}+\epsilon\right)$. Thus our stationary distribution proves to be a Negative Binomial with $p$ degrees of freedom and mean $\epsilon / \epsilon_{0}=c /(1-c)$

$$
P_{0}(i)=\frac{1}{\Gamma(p)}(i+1)_{p-1}(1-c)^{p} c^{i}
$$


The appropriate moments, which we shall also require later are the factorial moments defined

$$
\bar{m}_{n}(\infty)=\sum_{i=n}^{\infty} i^{(n)} P_{0}(i)
$$

These may be evaluated for the above distribution by the binomial theorem to give

$$
\bar{m}_{n}(\infty)=(p)_{n}\left(\epsilon / \epsilon_{0}\right)^{n}=(p)_{n}\left(\frac{c}{1-c}\right)^{n} .
$$

The limiting process (3.19) whereby $P_{0}(i)$ is converted to $P_{0}(x) \mathrm{d} x$ is crucial to the interconnection of discrete and continuous models throughout this paper. Upon applying it we arrive again at the Gamma distribution (5.4) with mean $\epsilon$, i.e.

$$
P_{0}(x) \mathrm{d} x=\lim _{\left[\begin{array}{l}
i \rightarrow \infty, \epsilon_{0} \rightarrow 0 \\
i \epsilon_{0}=x
\end{array}\right]} P_{0}(i) \Delta i
$$

where $\Delta i=1$ in the discrete distribution is to be replaced by $\mathrm{d} x / \epsilon_{0}$ in the probability density.

As we earlier emphasized, a full treatment of equilibrium would involve a proof of the $\boldsymbol{H}$-theorem for the above kernels $\boldsymbol{W}_{p, p}$. Although straightforward, this exercise is superfluous in that a proof can be given for the whole class of kernels satisf ying microscopic reversibility. (See Hoare ${ }^{25}$ ).

\section{Fourier expansion of the general solution}

It should perhaps be stressed that very few nonlinear problems can indeed be solved by an expansion in a set of orthogonal polynomials. Hence it is remarkable that for a large class of Maxwell models, in which our continuous models are contained as a special case, the general solution ${ }^{8}$ ) can be given in the form of a series expansion in Laguerre polynomials for rather general initial conditions. The coefficients in this expansion, the so-called Laguerre or Sonine moments, satisfy the same recursive system of equations as the ordinary moments, which can be solved sequentially ${ }^{1}$ ).

In this paper we shall extend the results to our discrete models, following an alternative method to that used in ref. 8. For the sake of a self-contained presentation we shall discuss both continuous and discrete cases.

It is well known that recursive systems for the calculation of moments of the unknown probability distribution can often be arrived at by direct integration of the Boltzmann equation and it is no surprise that the method 
works with the simple models discussed here. It is likewise common knowledge that the moment problem - of reconstructing the required distribution from its moments - can be circumvented if, instead of determining the simple moments, we are able to find appropriate polynomial moments, in terms of which a Fourier resolution of the desired function can be written. The choice of polynomials to be used falls naturally upon the set orthogonal with respect to the equilibrium distribution as weight function.

We shall consider next various aspects of the moment behaviour for the continuous and discrete models, again emphasizing the latter because of its relative unfamiliarity. The results, as well as giving general insight, play an important part in the systematic comparison of alternative solutions, whether of similarity type, or arising through linearization or other approximations.

\subsection{Moments for the continuous Beta model}

\subsubsection{Ordinary moments}

In this the simplest of cases we treat it is a straightforward matter to obtain the relevant moment evolution equations. Multiplying the kinetic equation (4.1) by $x^{n} / n$ ! and integrating we obtain,

$$
\left(\frac{\mathrm{d}}{\mathrm{d} t}+1\right) \mu_{n}(t)=\frac{1}{n !} \int_{0}^{\infty} \mathrm{d} u \int_{0}^{\infty} \mathrm{d} y P(y, t) P(u, t) \int_{0}^{u+y} x^{n} W_{p, p}(x, u+y) \mathrm{d} x .
$$

Since the inner integral is just the moment (3.11) of the symmetrical Beta distribution, i.e. in this case $(u+y)^{n}(p)_{n} /(2 p)_{n}$, we find, on applying the binomial theorem and cancelling factorials

$$
\left(\frac{d}{d t}+1\right) \mu_{n}(t)=\frac{(p)_{n}}{(2 p)_{n}} \sum_{k=0}^{n} \mu_{k}(t) \mu_{n-k}(t)
$$

It is a simple matter to show that the above is satisfied, for $t \rightarrow \infty$, by the Gamma distribution moments $\mu_{n}(\infty)=(p)_{n} \epsilon^{n} / n !$ !. Thus the stationary character of this distribution is confirmed. A more painstaking analysis similar to that of Ray ${ }^{18}$ ) can be used to show that the same is reached asymptotically - but this is superfluous and a far simpler method is available, as we shall demonstrate in the next section.

In the special case $p=1$ the factor on the right reduces to $1 /(n+1)$ and the equation becomes identical with that obtained by Krook and $\mathrm{Wu}$ for the moments of their Maxwell molecule model.

The most important feature of the above equations is of course that each $\mu_{n}(t)$ can, in principle, be found by recurrence from one order to the next, 
only moments of index $k<n$ being required to determine $\mu_{n}$. While the lower orders of $\mu_{n}(t)$ can be found relatively simply in explicit form, e.g. by a Laplace transform method, this process rapidly becomes tedious. We shall quote only the formulae for the second and third moments:

$$
\begin{aligned}
& \Delta \mu_{2}(t)=\Delta \mu_{2}(0) \mathrm{e}^{-\Lambda_{2} t}, \\
& \Delta \mu_{3}(t)=\Delta \mu_{3}(0) \mathrm{e}^{-\Lambda_{3} t}+\frac{3 \mu_{3}(\infty)}{\mu_{2}(\infty)} \Delta \mu_{2}(0)\left[\mathrm{e}^{-\Lambda_{2} t}-\mathrm{e}^{-\Lambda_{3} t}\right]
\end{aligned}
$$

with $\Delta \mu_{n}(t)=\mu_{n}(t)-\mu_{n}(\infty)$ and $\Lambda_{n}=1-2(p)_{n} /(2 p)_{n}$. Furthermore

$$
\begin{aligned}
& \mu_{1}=p \epsilon \text { for all } t, \\
& \mu_{2}(\infty)=\frac{1}{2} p(p+1) \epsilon^{2}, \\
& \mu_{3}(\infty)=\frac{1}{6} p(p+1)(p+2) \epsilon^{3} .
\end{aligned}
$$

\subsubsection{Laguerre (Sonine) moments}

The polynomials orthogonal with respect to the Gamma distribution (5.4) as weight function are the Laguerre set $L_{n}^{(p-1)}(x / \epsilon)$ which, traditionally called Sonine polynomials and used with $p=3 / 2$, are well known in the gas-kinetic theory of the Boltzmann equation ${ }^{26}$ ). The required properties are listed in an appendix.

In order to apply the method of Sonine moments in the present problem we use the completeness relation $\left(A 4^{\prime \prime}\right)$ and arrive at a Fourier expansion of the distribution $P(x, t)$ in the form

$$
P(x, t)=P_{0}(x) \sum_{n=0}^{\infty} \gamma_{n}(t) L_{n}^{(p-1)}(x / \epsilon) n ! /(p)_{n}
$$

with Sonine moments $\gamma_{n}(t)$ defined by the integrals

$$
\gamma_{n}(t)=\int_{0}^{\infty} L_{n}^{(p-1)}(x / \epsilon) P(x, t) \mathrm{d} x
$$

To obtain moment equations we multiply both sides of (4.1) by $L_{k}^{(p-1)}(x / \epsilon)$ and integrate to obtain an evolution equation identical with (6.1) except in having the polynomial instead of $x^{n} / n !$ in the inner integral. Using (A.8 ) with $p=q$ we arrive at the equation

$$
\left(\frac{\mathrm{d}}{\mathrm{d} t}+1\right) \gamma_{n}(t)=\frac{(p)_{n}}{(2 p)_{n}} \int_{0}^{\infty} \mathrm{d} y P(y) \int_{0}^{x} \mathrm{~d} u P(u) L_{n}^{(2 p-1)}((u+y) / \epsilon) .
$$

The trick is now to use the addition formula (A.6') for the Laguerre polynomials in the equation above, which leads to a convolution of moments on 
the right. Thus we obtain

$$
\left(\frac{\mathrm{d}}{\mathrm{d} t}+1\right) \gamma_{n}(t)=\frac{(p)_{n}}{(2 p)_{n}} \sum_{k=0}^{n} \gamma_{k}(t) \gamma_{n-k}(t)
$$

and find that the evolution equation satisfied by the Sonine moments is precisely identical in form to that for the ordinary moments. It follows that any algorithm for solving the above set is equally applicable in either case given of course that the boundary conditions will be different for $\mu_{n}$ and $\gamma_{n}$. Concerning the initial conditions, we may note that, whereas $\mu_{0}=1$ and $\mu_{1}=p \epsilon, \gamma_{0}=1$ and $\gamma_{1}=0$ for all $t$. The latter follows immediately from the fact that $L P^{(p-1)}(x / \epsilon)=p-x / \epsilon$. The time dependence of the Sonine moments is altogether simpler than that of the $\mu_{n}(t)-$ e.g. $\gamma_{2}(t)=\gamma_{2}(0) \exp \left(-\Lambda_{2} t\right)$. Most important, however, is the fact that, given this result the time-dependence of all Sonine moments with $n \geqslant 2$ can be written as a sum of pure exponential transients without constant terms. Thus not only is the equilibrium distribution determined by the fact that $\gamma_{n}(\infty)=\delta_{n 0}$ but the approach to it under the moment equations becomes self-evident. In this way the effect of the $\boldsymbol{H}$-theorem is made explicit and we can bypass altogether the tedious analysis given by Ray $^{18}$ ) to justify the same for the Krook-Wu model using only the ordinary moments.

\subsection{Moments of the discrete NHG model}

The development of moment equations for the discrete random scattering model is closely parallel to that set out for the continuous case. Our main innovation in this section is to explain the finite analogue of the Sonine moments which, as we shall show, are generated by the Meixner polynomials. The most striking result, however, is that, when suitably defined, both ordinary and polynomial discrete moments can be made to satisfy the same simple evolution equations as (6.2) and (6.8) in the continuous case.

\subsubsection{Ordinary factorial moments}

Defining reduced factorial moments $\bar{\mu}_{n}(t)=\bar{m}_{n}(t) / n$ ! with $\bar{m}_{n}(t)$ given by (4.4), we may derive a moment evolution equation by operating on both sides of (4.2) and summing, with the result that

$$
\begin{aligned}
\left(\frac{\mathrm{d}}{\mathrm{d} t}+1\right) \bar{\mu}_{n} & =\frac{(p)_{n}}{n !(2 p)_{n}} \sum_{j=0}^{\infty} \sum_{k=j}^{\infty}(k)^{(n)} P(j) P(k-j) \\
& =\frac{(p)_{n}}{(2 p)_{n} n !} \sum_{l=0}^{n}\left(\begin{array}{l}
n \\
l
\end{array}\right) \sum_{j=1}^{\infty}(j)^{(l)} P(j) \sum_{k=0}^{\infty}(k)^{(n-l)} P(k) .
\end{aligned}
$$


Note that in the last expression we have used Vandermonde's theorem (3.16) to expand the falling factorial binomial, after a shift $k \rightarrow k+j$ in the inner summation. On slight rearrangement it now becomes clear that

$$
\left(\frac{\mathrm{d}}{\mathrm{d} t}+1\right) \bar{\mu}_{n}(t)=\frac{(p)_{n}}{(2 p)_{n}} \sum_{=0}^{n} \bar{\mu}_{l}(t) \bar{\mu}_{n-l}(t),
$$

an equation again identical in form with that for the continuous model. Similar remarks to those made in section 6.1.1 apply here - we must only bear in mind that $\mu_{n}(t)$ and $\bar{\mu}_{n}(t)$ differ dimensionally by a factor $\epsilon_{0}^{n}$ which converts the state-occupancy probability to the energy probability density. Thus the equations for second and third moment evolution (6.3) and (6.4) need changing only by the substitution of $\epsilon / \epsilon_{0}$ for $\epsilon$ and addition of a bar over the $\mu$-symbols. The continuous limit may be taken at any stage in the above with the expected result, the connection between both moments being given by (4.5).

\subsubsection{Meixner moments of the NHG model}

In order to find the discrete moments analogous to the Sonine moments of the previous sections, we must turn to the polynomial set orthogonal over the positive integers with respect to the distribution (5.10) as weight-function. The required set are the Meixner polynomials $\left.\left\{M_{k}(i, p, c)\right\}^{27.28}\right)$ with $c=\epsilon /\left(\epsilon+\epsilon_{0}\right)$ as in the stationary distribution (5.10), and its properties are given in an appendix.

We may use the completeness relation (A.4) to put the probability distribution in the form

$$
P(i, t)=P_{0}(i) \sum_{n=0}^{\infty} \bar{\gamma}_{n}(t) M_{n}(i, p, c)
$$

with $P_{0}(i)$ the equilibrium distribution (5.10). We define the Meixner moments of the distribution $P(i, t)$ as

$$
\bar{\gamma}_{n}(t)=c^{n} \frac{(p)_{n}}{n !} \sum_{i=0}^{\infty} M_{n}(i, p, c) P(i, t) .
$$

Evidently $\bar{\gamma}_{0}=1$ by the normalization (4.6). The result of multiplying the kinetic equation by $c^{n}(p)_{n} / n$ ! and summing is then that

$$
\left(\frac{\mathrm{d}}{\mathrm{d} t}+1\right) \bar{\gamma}_{n}=c^{n} \frac{(p)_{n}}{n !} \sum_{k=0}^{\infty} \sum_{j=0}^{k} P(j) P(k-j) \sum_{i=0}^{k} M_{n}(i, p, c) W_{p, p}(i, k) .
$$

By application of (A.8) with $p=q$ we thus arrive at the equation

$$
\left(\frac{\mathrm{d}}{\mathrm{d} t}+1\right) \bar{\gamma}_{n}=c^{n} \frac{(p)_{n}}{n !} \sum_{k=0}^{\infty} \sum_{j=0}^{k} P(j) P(k-j) M_{n}(k, 2 p, c) .
$$


We have only to insert (A.6) with $p=q$ into the right-hand side of $(6.14)$ to see that

$$
\left(\frac{\mathrm{d}}{\mathrm{d} t}+1\right) \bar{\gamma}_{n}(t)=\frac{(p)_{n}}{(2 p)_{n}} \sum_{k=0}^{n} \bar{\gamma}_{k}(t) \bar{\gamma}_{n-k}(t)
$$

Thus the rate equation satisfied by the Meixner moments is identical in form to that for the ordinary moments of the same model, eq. (6.10), and also identical to that for the Sonine moments for the corresponding continuous problem with the Beta scattering kernel (6.8). The acceptable solutions to these equations must again satisfy $\bar{\gamma}_{0}(t)=1$ (number conservation) and $\bar{\gamma}_{1}(t)=$ 0 (energy conservation). The approach to the stationary solution $\gamma_{n}(\infty)=\delta_{n 0}$ can be discussed in a similar way.

Just as with the better-known Sonine moments, possession of the Meixner moments by solution of the above equations leads to an immediate solution of the moment problem for the recovery of $P(i, t)$-as is, of course, not possible when only the ordinary moments $\mu_{n}$ are available. The class of functions so recoverable will be those within the Hilbert space $l_{2}[0, \infty]$, the space of square-summable functions defined on the positive integers. This (unlike the case of $L_{2}$ functions of the continuous variable) is adequate for all applications where $P(i, t)$ is of the nature of a probability distribution.

\section{Linearization approximations}

The last topic we shall investigate will be the linearization of the transport equations (4.1) and (4.2) in the random scattering models. The use of linearized forms in the kinetic-theory Boltzmann equation has provided an extensive and sometimes controversial literature; only in extremely rare cases, such as we have here, has it ever been possible to effect a definite comparison between an exact solution and its linearized counterpart.

To economize our presentation, however, we shall concentrate almost entirely upon the discrete NHG model, afterwards obtaining the corresponding results for the continuous variable by taking the appropriate limit. It will be a simple matter for the reader, having studied the previous sections, to fill in the detailed derivation for the continuous case by simple analogy.

\subsection{Linearization of the discrete NHG model}

For the case of random scattering considered here, the linearization of the kinetic equation (4.2) is quite straightforward. It is however simpler to perform the linearization in a different representation, namely in the space of 
the Meixner-polynomials. We recall that the Meixner-moments $\bar{\gamma}_{n}(t)$ obey eq. (6.15) and approach the stationary solution $\bar{\gamma}_{n}(\infty)=\delta_{n, 0}$. In order to linearize around $\bar{\gamma}_{n}(\infty)$ we introduce the deviations:

$$
b_{n}(t) \equiv \bar{\gamma}_{n}(t)-\bar{\gamma}_{n}(\infty)
$$

which are assumed to be small. On inserting this into (6.15) and neglecting second order small quantities we see that the $b_{n}(t)$ obey the linearized moment-equations:

$$
\left(\frac{\mathrm{d}}{\mathrm{d} t}+\Lambda_{n}\right) b_{n}(t)=0
$$

where

$$
\Lambda_{n}=1-2(p)_{n} /(2 p)_{n}
$$

are the eigenvalues of the linearized Boltzmann equation. The solution of (7.2) is given by

$$
b_{n}(t)=b_{n}(0) \mathrm{e}^{-\Lambda_{n} t}
$$

Thus the solution to the linearized Boltzmann equation is, according to (6.11), given by

$$
P^{L}(i, t)=P_{0}(i)\left\{1+\sum_{k=2}^{\infty} b_{k}(0) \mathrm{e}^{-A_{k^{t}}} M_{k}(i, p, c)\right\}
$$

where the constants $b_{k}(0)$ are simply the Meixner moments of the initial distribution $P(i, 0)$. Note that energy conservation implies that $b_{1}(0)$ vanishes.

For completeness we will give the form of the linearized Boltzmann equation for the distribution function, which can be obtained by inserting:

$$
P^{\mathrm{L}}(i, t)=P_{0}(i)+h(i, t)
$$

into eq. (4.2). Here $P_{0}(i)$ is the Negative Binomial distribution (5.10) and $h(i, t)$ denotes the deviation from equilibrium. The equation for $h$, after neglecting terms of order $h^{2}$, reads

$$
\left(\frac{\partial}{\partial t}+1\right) h(i, t)=2 \sum_{j=0}^{\infty} K(i, j) h(j, t),
$$

where the kernel is given by

$$
\begin{aligned}
K(j, i) & =\sum_{k=\max (i, j)}^{\infty} P_{0}(k-i) W_{p, p}(j, k) \\
& =\frac{\Gamma(2 p)}{\Gamma^{3}(p)} \sum_{k=\max (i, j)} \frac{(j+1)_{p-1}(k-j+1)_{p-1}(k-i+1)_{p-1}}{(k+1)_{2 p-1}} c^{k-i}(1-c)^{p} .
\end{aligned}
$$


What we have shown in fact is that the right eigenfunctions associated with the eigenvalue $\frac{1}{2}\left(1-\Lambda_{k}\right)=(p)_{k} /(2 p)_{k}$ are $P_{0}(i) M_{k}(i, p, c)$ where $M_{k}(i, p, c)$ are the Meixner polynomials. This result has already been obtained by Hoare and Rahman ${ }^{15}$ ), who studied a somewhat more general form of the kernel $K(j, i)$.

We observe finally that the complete and linearized equations for the Meixner moments $\bar{\gamma}_{2}(t)$ and $\bar{\gamma}_{3}(t)$ are identical. This implies that for long times in a finite range of energies the full distribution function $P(x, t)$ is approximated by $P^{\mathrm{L}}(x, t)$ up to a correction of order $\mathcal{O}\left(\mathrm{e}^{-\Lambda_{4} t}\right)$.

\subsection{Linearization of the continuous model}

For the continuous model, the equations for the Sonine moments are exactly the same as those for the Meixner moments in the discrete case, so we can simply copy the solution

$$
b_{n}(t) \equiv \gamma_{n}(t)-\gamma_{n}(\infty)=b_{n}(0) \mathrm{e}^{-A_{n} t}
$$

and the linearized solution in energy space can thus be written

$$
P^{\mathrm{L}}(x, t)=P_{0}(x)\left\{1+\sum_{k=2}^{\infty} b_{k}(t) L_{k}^{(p-1)}\left(\frac{x}{\epsilon}\right)\right\} .
$$

The corresponding linearized Boltzmann equation for the energy distribution function reads in this case

$$
\left(\frac{\partial}{\partial t}+1\right) h(x, t)=2 \int_{0}^{\infty} K(x, y) h(y, t) \mathrm{d} y .
$$

The kernel is now given by

$$
\begin{aligned}
K(y, x) & =\int_{u=\max (x, y)}^{\infty} P_{0}(u-x) W_{p, p}(y, u) \mathrm{d} u \\
& =\frac{\Gamma(2 p)}{\Gamma^{3}(p)} \int_{u=\max (x, y)}^{\infty} \frac{[y(u-y)(u-x)]^{p-1}}{u^{2 p-1}} \frac{\mathrm{e}^{-(u-x) / \epsilon}}{\epsilon^{p}} \mathrm{~d} u
\end{aligned}
$$

where $P_{0}(x)$ is the Gamma-distribution (5.4). This is also a special case of a kernel treated by Cooper and Hoare $\left.{ }^{15 a}\right)$. Its eigenfunctions are $P_{0}(x) L_{k}^{p-1}(x / \epsilon)$ with again the same eigenvalues $\frac{1}{2}\left(1-\Lambda_{k}\right)$ : a result also obtained in $[2,8]$.

\section{Appendix}

We discuss the required properties of Meixner polynomials, and obtain the corresponding properties for Laguerre polynomials by taking the continuous limit (3.19). 
The Meixner polynomials can be defined in various ways, in particular

$$
\begin{aligned}
M_{k}(i, p, c) & ={ }_{2} F_{1}\left(-k,-i, p ; 1-c^{-1}\right) \\
& =\sum_{\nu=0}^{\operatorname{ming}(i, k)} \frac{(-k)_{\nu}(-i)_{\nu}\left(1-c^{-1}\right)^{\nu}}{(p)_{\nu} \nu !}
\end{aligned}
$$

and the continuous-variable limit, yielding the Laguerre polynomials is

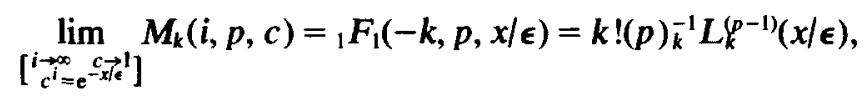

where we used the relation

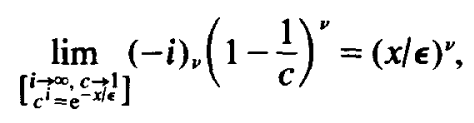

so that

$$
L_{k}^{(p-1)}(x)=\sum_{\nu=0}^{k} \frac{(-x)^{\nu}}{\nu !}\left(\begin{array}{c}
k+p-1 \\
k-\nu
\end{array}\right)
$$

In our present context the limit $c \rightarrow 1$ will be seen to be equivalent to letting the quantum size tend to zero, $\epsilon_{0} \rightarrow 0$. This is, of course, the same limit that takes the NHG distribution (3.13) into the Beta distribution (3.9) and the Negative Binomial (5.10) into the Gamma distribution (5.4). The orthogonality sum is

$$
c^{k} \frac{(p)_{k}}{k !} \sum_{i=0}^{\infty} P_{0}(i) M_{k}(i, p, c) M_{i}(i, p, c)=\delta_{k l}
$$

with $P_{0}(i)$ defined in (5.10). They have the important property of self-duality, which is to say that $M_{k}(i, .,)=.M_{i}(k, .,$.$) . Rewritten with this interchange, the$ above sum becomes a completeness relation, i.e. an explicit expansion of the Kronecker delta-function. Replacing $i=x / \epsilon_{0}$ and taking the continuous limit we obtain the orthogonality relation for the Laguerre polynomials

$$
\int_{0}^{\infty} P_{0}(x) L_{k}^{(p-1)}\left(\frac{x}{\epsilon}\right) L^{(p-1)}\left(\frac{x}{\epsilon}\right) \mathrm{d} x=\frac{(p)_{k}}{k !} \delta_{k l} .
$$

From (A.4) we can formally obtain in a similar way the completeness relation for the Laguerre polynomials

$$
P_{0}(x) \sum_{n=0}^{\infty} \frac{n !}{(p)_{n}} L_{n}^{(p-1)}\left(\frac{x}{\epsilon}\right) L_{n}^{(p-1)}\left(\frac{y}{\epsilon}\right)=\delta(x-y),
$$

where $\delta(x-y)$ is the Dirac delta function. 
Other properties which we shall require are the generating function

$$
\frac{(1-s)^{p}}{\Gamma(p)} \sum_{i=0}^{\infty}(i+1)_{p-1} s^{i} M_{k}(i, p, c)=\left(\frac{1-s / c}{1-s}\right)^{k}
$$

and its continuous limit

$$
(1-s)^{p} \sum_{n=0}^{\infty} s^{n} L_{n}^{(p-1)}(x / \epsilon)=\exp \left[-\frac{x s}{\epsilon(1-s)}\right]
$$

In the formulae for the Meixner polynomials the subscript and the variable may be interchanged at will. We further need the addition formula

$$
M_{k}(n, p+q, c)=\sum_{i=0}^{n} W_{q, p}(l, n) M_{k-j}(n-l, p, c) M_{j}(l, q, c)
$$

with $j=0,1, \ldots, k$, and

$$
W_{q, p}(l, n)=\frac{(l+1)_{q-1}(n-l+1)_{p-1}}{B(q, p)(n+1)_{q+p-1}}
$$

is the Negative Hypergeometric distribution. The proof follows by using the generating function (A.5) with $p \rightarrow p+q$; then factoring its right hand side $A^{k}$ into $A^{k-j} \cdot A^{j}$; next applying (A.5) to the first factor with $k \rightarrow k-j$, and to the second with $p \rightarrow q$ and $k \rightarrow j$, and finally equating the coefficients of equal powers of $s$ in the resulting series. We require also the special case $j=k$ with $M_{0}(k, p, c)=1$ in (A.6), and by using the self-duality property we obtain

$$
M_{k}(n, p+q, c)=\sum_{i=0}^{k} W_{q, p}(i, k) M_{n}(i, q, c) .
$$

This formula, which is the discrete analogue of the Kogbetliantz formula, was first given by Cooper, Hoare and Rahman ${ }^{15}$ ). In the continuous limit with $k=(u+y) / \epsilon_{0}$ and $j=u / \epsilon_{0}$ and again using the self-duality property equation (A.8) becomes the addition formula for Laguerre polynomials

$$
L_{n}^{(p+q-1)}((u+y) / \epsilon)=\sum_{l=0}^{n} L_{n-l}^{(p-1)}(y / \epsilon) L^{(q-1)}(u / \epsilon),
$$

whereas (A.8) yields with $k=u / \epsilon_{0}$ and $i=x / \epsilon_{0}$ the Kogbetliantz formula [ref. 28 , formula 10.12 .30$]$

$$
\frac{(q)_{n}}{(p+q)_{n}} L_{n}^{(p+q-1)}(u / \epsilon)=\int_{0}^{u} \mathrm{~d} x W_{q, p}(x, u) L_{n}^{(q-1)}(x / \epsilon) .
$$

The kernel is the Beta distribution

$$
W_{q, p}(x, u)=\frac{x^{q-1}(u-x)^{p-1}}{B(q, p) u^{q+p-1}}
$$


We may note the curious property that the NHG and Beta distributions (A.7), (A.7') are respectively the kernels of $p$-step sum and integral ladder-operators acting on the relevant parameters of the Meixner and Laguerre polynomial sets.

\section{References}

1) C. Truesdell, J. Rational Mech. Anal. 5 (1956) 55.

2) A.V. Bobylev, Sov. Phys. Dokl. 20 (1976) 820, 822.

3) A.V. Bobylev, Sov. Phys. Dokl. 21 (1976) 632.

4) M. Krook and T.T. Wu. Phys. Rev. Lett. 36 (1976) 1107;

Physics of Fluids 20 (1977) 1589.

5) G. Tenti and W.H. Hui, J. Math. Phys. 19 (1978) 774.

6) J.A. Tjon and T.T. Wu, Phys. Rev. A19 (1979) 883.

7) J.A. Tjon, Phys. Lett. 70A (1979) 369.

8) M.H. Ernst, Phys. Lett. 69A (1979) 390.

9) M.H. Ernst, in Proc. of the Conf. on Mathematical Problems in the Kinetic Theory of Gases, May 20-26, 1979 at Math. Forschungsinstitut, Oberwolfach, W. Germany, H. Neunzert, ed.

10) M. Kac, in: Proceedings III Berkeley Symposium on Mathematics, Statistics and Probability (Univ, of California Press, Berkeley, 1955) p. 171.

11) M. Barnsley and G. Turchetti, Phys. Lett. 72A (1979) 417.

12) M. Barnsley and H. Cornille, CEN-Saclay preprint DPh-T/79/73, May 1979; Dth-T/79/79; June 1979.

13) H. Cornille, CEN-Saclay preprint DTh-T/79/102, July 1979.

14) U. Weinert, S.L. Liu and E.A. Mason, preprint, Brown University, June 1979.

15) (a) R.D. Cooper and M.R. Hoare, J. Stat. Phys. 20 (1979) 597;

(b) R.D. Cooper, M.R. Hoare and M. Rahman, J. Math. Anal. \& Appns 61 (1977) 262;

(c) M.R. Hoare and M. Rahman, Physica 97A (1979) 1; E. Futcher and M.R. Hoare, to be published.

16) M.H. Ernst and E.M. Hendriks, Phys. Lett. 70A (1979) 183.

17) S. Rouse and S. Simons, J. Phys. A9 (1976) L155; A11 (1978) 423.

18) D. Ray, J. Stat. Phys. 20 (1979) 115.

19) K.E. Shuler, J. Chem. Phys. 32 (1960) 1692.

20) C.C. Rankin and J.C. Light, J. Chem. Phys. 46 (1967) 1305.

21) K. Koura, Rarefied Gas Dynamics, K. Karamechi, ed., 8th Intern. Symp., Stanford (1972) (Academic Press, New York, 1974) p. 487.

22) Z.A. Melzak, Trans. Am. Math. Soc. 85 (1957) 547.

23) L. Waldmann, in Handbuch der Physik, S. Flügge, ed., Vol. 12 (Springer Verlag, Berlin, 1958).

24) H.P. McKean, Jr., J. of Comb. Theory 2 (1967) 358.

25) M.R. Hoare, to be published.

26) S. Chapman and T.G. Cowling, The Mathematical Theory of Non-uniform Gases, 3rd edition (Cambridge at the Univ. Press, 1970).

27) J. Meixner, J. Lond. Math. Soc. 9 (1934) 6.

28) A. Erdelyi, (ed.), Higher Transcendental Functions (McGraw-Hill, New York, 1953) Vol. 2, Section 10.24. 